\title{
Mixed Tunnel-FET/MOSFET Level Shifters: a new proposal to extend the Tunnel-FET application domain
}

\author{
Marco Lanuzza, Member, IEEE, Sebastiano Strangio, Felice Crupi, Senior Member, IEEE, Pierpaolo \\ Palestri, Senior Member, IEEE, and David Esseni, Fellow, IEEE
}

\begin{abstract}
In this work, we identify the level shifter for voltage up-conversion from the ultralow voltage regime as a key application domain of tunnel field-effect transistors. We propose a mixed TFET-MOSFET level shifter design methodology, which exploits the complementary characteristics of TFET and MOSFET devices. Simulation results show that the hybrid level shifter exhibits superior dynamic performance at the same static power consumption compared to conventional MOSFET and pure TFET solutions. The advantage of the mixed design with respect to the conventional MOSFET approach is emphasized when lower voltage signals have to be up-converted, reaching an improvement of the energy delay product up to 3 decades. When compared to the full MOSFET design, the mixed TFETMOSFET solution appears to be less sensitive towards threshold voltage variations in terms of dynamic figures of merit, at the expense of higher leakage variability. Similar results are obtained for four different level shifter topologies, thus indicating that the hybrid TFET-MOSFET approach offers intrinsic advantages in the design of level shifter for voltage up-conversion from the ultralow voltage regime compared to conventional MOSFET and pure TFET solutions.
\end{abstract}

Index Terms- Tunnel field-effect transistor (TFET), level shifter (LS), technology computer aided design (TCAD).

\section{INTRODUCTION}

$\mathrm{W}$ ITH the growing interest in low energy-budget electronic applications, the tunnel field-effect transistor (TFET) is playing a major role as a new device concept, featuring a better performance/leakage tradeoff than conventional MOSFET at scaled power supply voltage $\left(\mathrm{V}_{\mathrm{DD}}\right)$ levels. Differently from MOSFETs, where the minimum subthreshold swing (SS) is theoretically constrained to the 60

Manuscript received July 31, 2015. This work was supported in part by the European Community's Seventh Framework Program under Grant Agreement 619509 (Project E2SWITCH).

M. Lanuzza and F. Crupi are with the DIMES (Dipartimento di Ingegneria Informatica, Modellistica, Elettronica e Sistemistica), Università della Calabria, Rende 87036, Italy (email: lanuzza@dimes.unical.it, felice.crupi@unical.it).

S. Strangio is with the DIEGM (Dipartimento di Ingegneria Elettrica, Gestionale e Meccanica), Università degli Studi di Udine, Via delle Scienze 206, 33100 Udine, Italy and the DIMES (Dipartimento di Ingegneria Informatica, Modellistica, Elettronica e Sistemistica), Università della Calabria, Rende 87036, Italy (email: seb88str@gmail.com, sstrangio@dimes.unical.it).

P. Palestri and D. Esseni are with the DIEGM (Dipartimento di Ingegneria Elettrica, Gestionale e Meccanica), Università degli Studi di Udine, Via delle Scienze 206, 33100 Udine, Italy (e-mail: pierpaolo.palestri@uniud.it, david.esseni@uniud.it).
$\mathrm{mV} /$ decade limit at room temperature, TFETs can offer steeper turn-on characteristics due to Band-To-Band Tunneling (BtBT) current [1]. Several mixed device-circuit studies as well as device-circuit co-design strategies have investigated the potentialities of such device concept at both device and circuit levels [2-13].

In today complex system on chips (SoCs), the multi-supply voltage domain (MSVD) technique [14] is emerging as an effective approach to improve energy efficiency. The MSVD technique consists of partitioning the design into separate voltage domains (or "voltage islands"), each operating at a proper supply voltage level depending on its timing requirements. Time-critical domains run at higher power supply voltage $\left(\mathrm{V}_{\mathrm{DDH}}\right)$ to maximize the speed, whereas noncritical sections work at lower supply voltage $\left(\mathrm{V}_{\mathrm{DDL}}\right)$ to optimize energy consumption, thus effectively managing tasks that require substantially different performances. Minimizing the delay and energy overhead of level conversion between different voltage domains is a key challenge in the design of effective multi-supply SoCs, becoming particularly critical when the number of power domains and/or the data width in the SoC increase [15]. Within this context, several level shifter (LS) circuit topologies were recently proposed for speed- and energy-efficient wide-range conversion from the deep subthreshold regime up to the nominal supply voltage level [1621].

In this work, we propose a mixed TFET-MOSFET LS design methodology, which exploits the complementary characteristics of TFETs and MOSFETs for voltage upconversion. Among several mixed device/circuit works, only few of them have proposed and investigated mixed TFETMOSFET solutions (e.g. hybrid SRAM cells [10,11]). Our work hinges in this context with the aim of identifying an additional area of application where the mixed TFETMOSFET design can be an added value.

The remainder of the paper is organized as follows. Section II discusses the design and the main characteristics of the devices considered in this work. Section III introduces the analyzed LS circuit topologies alongside with the adopted simulation methodology. Comparative simulation results are discussed in Section IV. Finally, Section V concludes the paper. 

IEEE. Personal use is permitted, but republication/redistribution requires IEEE permission. See http://www.ieee.org/publications_standards/publications/rights/index.html for more information.

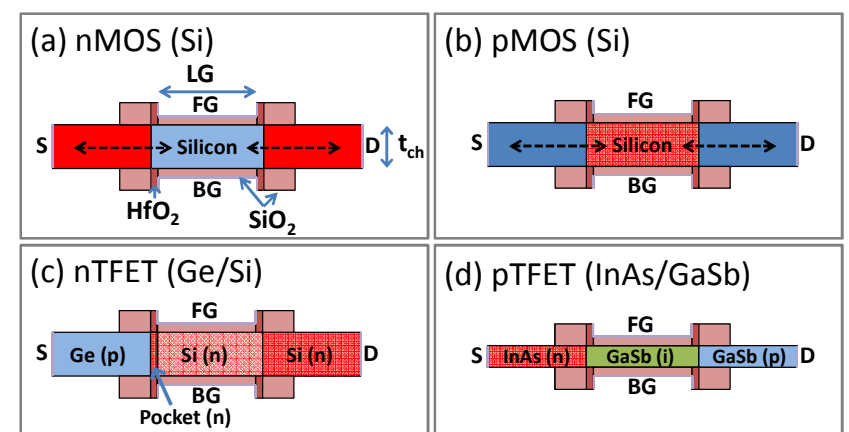

Fig.1. Structures of n-type MOSFET (a), p-type MOSFET (b), n-type TFET (c) and p-type TFET (d). Doping levels: MOSFETs: $\mathrm{N}_{\text {Source/Drain }}=10^{20} \mathrm{~cm}^{-3}$, $\mathrm{N}_{\text {Channel }}=10^{17} \mathrm{~cm}^{-3}$. nTFET: $\mathrm{N}_{\text {Source }}=2 \cdot 10^{19} \mathrm{~cm}^{-3}, \mathrm{~N}_{\text {Pocket }}=5 \cdot 10^{18} \mathrm{~cm}^{-3}$, $\mathrm{N}_{\text {Channel }}=10^{17} \mathrm{~cm}^{-3}, \mathrm{~N}_{\text {Drain }}=10^{19} \mathrm{~cm}^{-3} \cdot$ pTFET: $N_{\text {Source }}=2 \cdot 10^{18} \mathrm{~cm}^{-3}$, intrinsic channel, $\mathrm{N}_{\text {Drain }}=10^{19} \mathrm{~cm}^{-3}$. Dimensions: $\mathrm{L}_{\mathrm{G}}=30 \mathrm{~nm} . \mathrm{t}_{\mathrm{Ch}}=10 \mathrm{~nm}$ (except $\mathrm{t}_{\mathrm{Ch}}=5 \mathrm{~nm}$ for the InAs/GaSb pTFET).

\section{DEVICE DESIGN AND DEVICE LEVEL MODELS}

Ideal double-gate SOI geometries designed in [8] are used in this work as a reference for the conventional MOSFET devices, featuring a SS close to the $60 \mathrm{mV} / \mathrm{dec}$ theoretical limit. As a counterpart, hetero-junction TFETs featuring a SS below the $60 \mathrm{mV} / \mathrm{dec}$ have been designed. The same structure of MOSFETs (double-gate SOI with $\mathrm{L}_{\mathrm{G}}=30 \mathrm{~nm}$ and EOT = $1.1 \mathrm{~nm}$ ) was taken as a base in the definition of complementary TFETs, while the choice of the hetero-structure materials and channel thickness was driven by some constraints imposed by the particular circuit application, as will be shortly discussed in the following.

It is well known that TFETs can outperform MOSFETs only at very low $\mathrm{V}_{\mathrm{DD}}\left(\mathrm{V}_{\mathrm{DD}}<0.4 \mathrm{~V}\right)$ [6-12], that is in circuit applications where they can take advantage of their steep transition from the off- to the on-state. Recent works [7,9,12], based on device simulations with a more accurate description of the device physics with respect to the TCAD modeling level, claimed that III-V materials provide the opportunity to achieve both steep turn-on operation and on-current comparable with MOSFET up to a $V_{D D}$ of $400 \mathrm{mV}$. $\mathrm{GaSb} / \mathrm{InAs}$ hetero-structure, in the presence of a suitable amount of transverse quantization (e.g. quantum wells with thickness $\sim 5 \div 7 \mathrm{~nm}$ ), has been proposed to implement both $\mathrm{n}$ and p-type TFET devices [12,13], possibly with a slight $\mathrm{Al}$ concentration in the $\mathrm{GaSb}$ (e.g. $\mathrm{Al}_{0.05} \mathrm{Ga}_{0.95} \mathrm{Sb}$ ) [7].

In the p-type TFET implementation, InAs is used for the source region, whereas $(\mathrm{Al}) \mathrm{GaSb}$ is employed in the channel and drain regions. Although the complementary heterostructure could be used to implement also the n-type TFET, due to relatively low band-gap of the InAs ( $0.5 \mathrm{eV}$ [4], including quantization effects), it features a strong ambipolar leakage as the $V_{D S}$ is increased [12,13]. Being a $V_{D S}$ up to $1 \mathrm{~V}$ a key requirement of the LSs here discussed, InAs/GaSb hetero-junction has been used only to implement the pTFET. For the nTFET, germanium $(\mathrm{Ge}) /$ silicon $(\mathrm{Si})$ hetero-junction is proposed. The low band-gap Ge is used in the source region to ensure large $\mathrm{BtBT}$ rates, whereas $\mathrm{Si}$ is employed in the
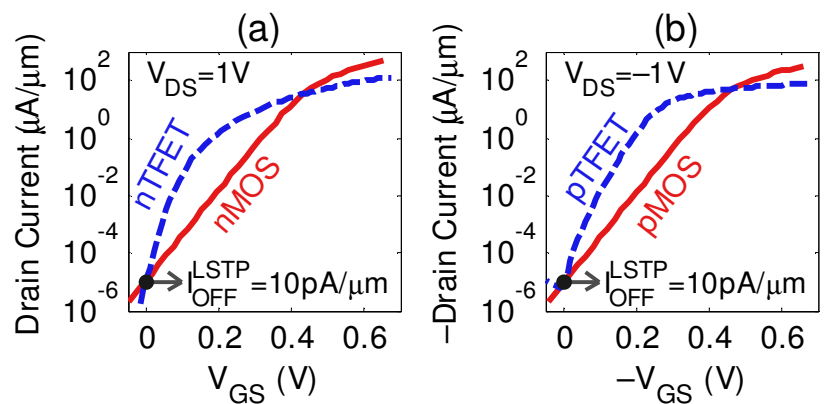

(c)
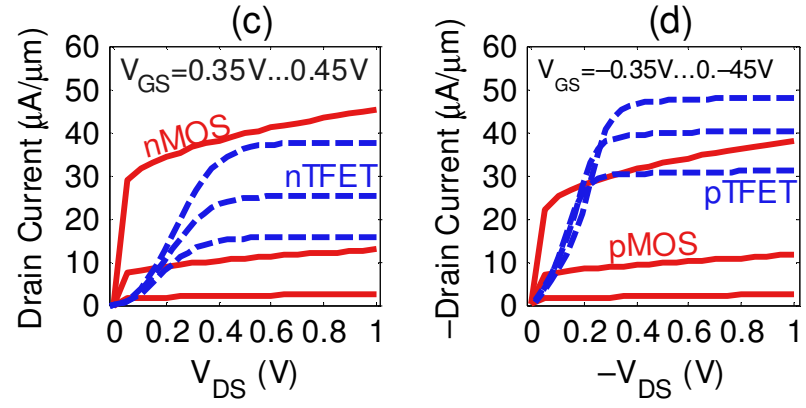

Fig.2. $\mathrm{I}_{\mathrm{D}}-\mathrm{V}_{\mathrm{GS}}$ characteristics at $\left|\mathrm{V}_{\mathrm{DS}}\right|=1 \mathrm{~V}$ of the considered n-type (a) and p-type (b) devices. $\mathrm{I}_{\mathrm{D}}-\mathrm{V}_{\mathrm{DS}}$ characteristics of the $\mathrm{n}$ - (c) and p-type (d) devices $\left(\left|\mathrm{V}_{\mathrm{GS}}\right|=0.35 \mathrm{~V}, 0.4 \mathrm{~V}, 0.45 \mathrm{~V}\right)$.

channel-drain regions to ensure a sufficient robustness against ambipolar leakage. Although the integration of III-V materials and Ge-on-Si CMOS substrate can be a challenging task, it has been already successfully explored in [22] with the integration of III-V nMOSFETs together with Ge pMOSFETs.

The TFET design has been performed with Sentaurus TCAD, starting from the reference MOSFET structure [8] and by choosing appropriate materials (and the related parameters) and doping levels to get complementary n- and p-TFET devices featuring sufficiently symmetric I-V characteristics. BtBT has been simulated with the dynamic non-local path BtBT model. Material parameters, such as the energy gap $\mathrm{E}_{\mathrm{G}}$ and electron affinity $\chi$, conduction and valence band density of states $\left(\mathrm{N}_{\mathrm{C}}{ }^{\mathrm{DOS}}\right.$ and $\mathrm{N}_{\mathrm{V}}{ }^{\mathrm{DOS}}$ ), BtBT model constants (Apath, Bpath, Rpath) have been calibrated against experimental data for the Ge-source nTFET [5] and against atomistic simulations for the InAs/GaSb pTFET $[4,13]$.

Materials and doping levels are reported in Fig.1 for all the considered devices. The thickness of the pTFET is set to $5 \mathrm{~nm}$, since the physical parameters [13] (band-gap, tunneling-rate, etc.) calibrated on the device published in [4], can be considered trustworthy only for a device with the same thickness.

Fig.2 show the I-V characteristics of the four devices depicted in Fig.1. In Fig.2a-b, the $\mathrm{I}_{\mathrm{D}}-\mathrm{V}_{\mathrm{GS}}$ characteristics are aligned - through a fine tuning of the metal gate work-function of any single device - to get the same normalized off-current, taking as target the value suggested by the ITRS for low standby power applications $\left(\mathrm{I}_{\mathrm{OFF}}{ }^{\mathrm{LSTP}}=10 \mathrm{pA} / \mu \mathrm{m}\right)$. For the chosen $\mathrm{I}_{\mathrm{OFF}}$, TFETs feature larger on-current than the corresponding MOSFETs up to a $\mathrm{V}_{\mathrm{GS}}$ of $\sim 400 \div 450 \mathrm{mV}$. Fig.2c-d shows the superliner output characteristics of TFETs along with the ones of the corresponding MOSFETs. The 

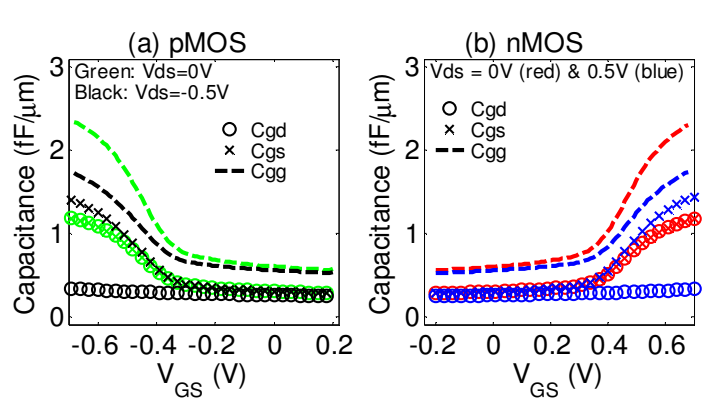

(c) pTFET
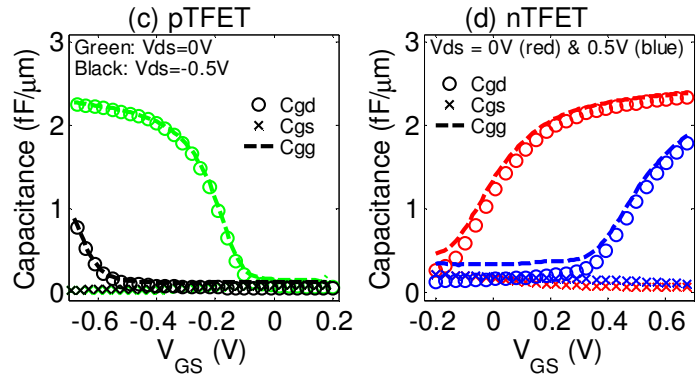

Fig.3. Gate capacitances versus $\mathrm{V}_{\mathrm{GS}}$ for the (a) p-type MOSFET, (b) n-type MOSFET, (c) p-type TFET and (d) n-type TFET

delayed turn-on behavior of TFETs is well known in the literature [2,3] and it has been shown that it degrades the inverter voltage-transfer-characteristic (VTC) and negatively impacts any CMOS-like logic gate [6-8]. Conversely, when the $\mathrm{V}_{\text {DS }}$ increases above $500 \mathrm{mV}$, TFET output characteristics show a flatter current than MOSFETs as a result of the higher robustness of the designed TFETs against the short-channel effects.

Capacitance characteristics are reported in Fig.3 for $\left|\mathrm{V}_{\mathrm{DS}}\right|=$ $0 \mathrm{~V}$ and $0.5 \mathrm{~V}$. Due to symmetric geometry, in MOSFETs the gate-to-source capacitance $\left(\mathrm{C}_{\mathrm{GS}}\right)$ and the gate-to-drain capacitance $\left(\mathrm{C}_{\mathrm{GD}}\right)$ are overlapped (for any $\mathrm{V}_{\mathrm{GS}}$ ) when $\mathrm{V}_{\mathrm{DS}}=0$ $\mathrm{V}$, whereas $\mathrm{C}_{\mathrm{GS}}$ becomes the main contribution to the overall gate capacitance $\left(\mathrm{C}_{\mathrm{GG}}\right)$ when the device is switching on (i.e. as $\mathrm{V}_{\mathrm{GS}}$ and $\mathrm{V}_{\mathrm{DS}}$ are increased). On the contrary, the TFET $\mathrm{C}_{\mathrm{GG}}$ is dominated by the $\mathrm{C}_{\mathrm{GD}}$. Interestingly, as $\mathrm{V}_{\mathrm{DS}}$ increases, the onset of the rising $\mathrm{C}_{\mathrm{GD}}$ with $\mathrm{V}_{\mathrm{GS}}$ is shifted toward larger $\mathrm{V}_{\mathrm{GS}}$ [8]. Due to convergence limits, the BtBT model was not activated in the $\mathrm{AC}$ analysis performed to generate the $\mathrm{C}_{\mathrm{GS}}$ and $\mathrm{C}_{\mathrm{GD}}$ characteristics. However, since the charge produced by the BtBT is not large enough to influence the device electrostatics (i.e. to modify the shape of the band diagrams), the impact of such simplification in the computed $\mathrm{C}_{\mathrm{GS}}$ and $\mathrm{C}_{\mathrm{GD}}$ is negligible.

\section{LEVEL SHIFTERS: BENCHMARKS AND SIMULATION METHODOLOGY}

In this Section, the LS circuit topologies referenced in this work are briefly discussed. After that, the circuit simulation methodology is detailed.

\section{A. Circuit Description}

Four of the most recent and efficient LS designs [18-21] were considered as case study. For each LS design the

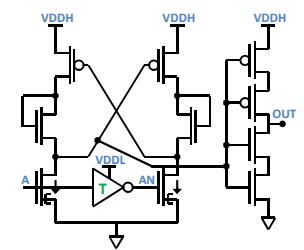

(a)

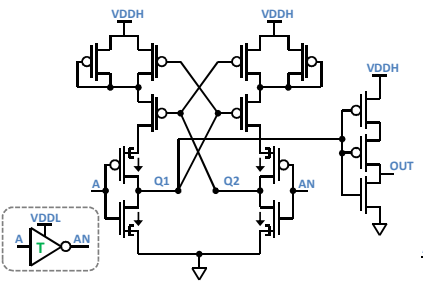

(c)

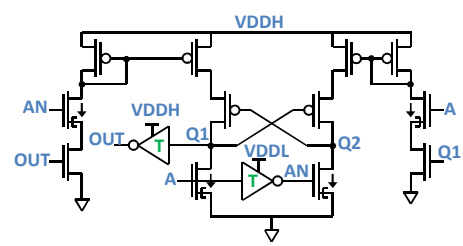

(b)

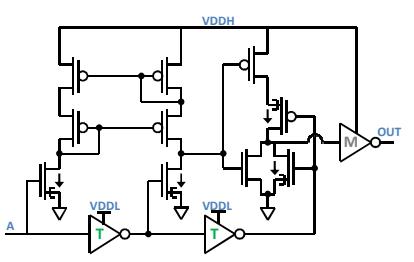

(d)

Fig.4. Mixed TFET-MOSFET LS design version of circuits reported in (a) [18], (b) [19], (c) [20] and (d) [21]. The "T" and "M" labels on the inverters mean all TFET and all MOSFET implementation, respectively. "A" and "AN" are the input signal and its inverted version.

conventional MOSFET, the pure TFET and the mixed TFETMOSFET (shown in Fig.4) implementations were considered. In the mixed TFET-MOSFET designs, only the MOSFETs directly driven by the low-voltage signals are replaced by TFET devices.

The key feature of the circuit proposed by Zhao et al. [18] (shown in Fig.4a) is the use of two NMOS-diodes to limit the current drawn by the opposing pull-up network (PUN) during the discharge of internal nodes (either Q1 or Q2). A similar achievement is obtained in the LS circuit proposed by Hosseini et al. [19] (depicted in Fig.4b) through the use of two current generators. The design proposed by one of the authors of this work [20] (shown in Fig.4c), exploits a different idea based on self-adapting PUNs to speed-up both high-to-low and low-to-high transitions of internal nodes Q1 and Q2. Finally, the circuit proposed by Luo et al. [21] (shown in Fig.4d), is based on a hybrid structure comprising a modified Wilson current mirror and a NOR CMOS logic gate to achieve effective voltage up-conversion while limiting stand-by power consumption.

\section{B. Simulation methodology}

Although it is possible to describe with the TCAD simulator simple circuit topologies implemented with few devices in the mixed device/circuit mode [11], the computation time becomes unacceptable when the number of devices increases up to approximately 10 (note that this number is strongly influenced by the complexity of the single device mesh and by models activated in the TCAD simulator). Furthermore, the model used in this work to take in account BtBT (i.e. the most physically accurate BtBT model available today in the TCAD simulator, the dynamic non-local path BtBT) shows poor convergence properties in the mixed-mode environment as well as in $\mathrm{AC}$ or transient simulations. For this reason, we have restricted the use of the TCAD simulator only to design the devices and to generate Look-Up Tables (LUT), in a defined 
(c) 2015 IEEE. Personal use of this material is permitted. Permission from IEEE must be obtained for all other users, including reprinting/ republishing this material for advertising or promotional purposes, creating new collective works for resale or redistribution to servers or lists, or reuse of any copyrighted components of this work in other works. DOI: 10.1109/TED.2015.2494845 - (C) 2015 IEEE. Personal use is permitted, but republication/redistribution requires IEEE permission. See http://www.ieee.org/publications_standards/publications/rights/index.html for more information.

range of $\mathrm{V}_{\mathrm{GS}}$ and $\mathrm{V}_{\mathrm{DS}}$, with drain current $\left(\mathrm{I}_{\mathrm{D}}\right)$ and capacitances $\left(\mathrm{C}_{\mathrm{GS}}\right.$ and $\left.\mathrm{C}_{\mathrm{GD}}\right)$ characteristics. Concerning the circuit level, Cadence has been used for time-efficient simulations by defining black-box devices in the Verilog-A description language. Such solution has allowed us also to perform (in a reasonable time) a statistical analysis of the effects at circuit level due to threshold voltage variability.

In our simulations, all the circuits are driven by an identical inverter in order to duly take into account the input gate capacitances of TFET and MOSFET devices. Additionally, output of the circuits drives a capacitance of $20 \mathrm{fF}$, which is a fairly conservative loading value, given that the input capacitance of a minimum-size inverter is $\sim 0.2 \mathrm{fF}$.

The referenced MOSFET designs have been sized to achieve the minimum energy delay product (EDP) for $0.3 \mathrm{~V}$ to $1 \mathrm{~V}$ voltage conversion and considering a $500 \mathrm{kHz}$ input pulse. In TFET-based circuits, TFET devices maintain the same sizes of replaced MOSFETs to assure similar leakage targets (as discussed in Section II, we set the same $\mathrm{I}_{\mathrm{OFF}}$ for both MOSFETs and TFETs).

\section{RESULTS AND DISCUSSION}

This Section presents the simulation results performed on the various LS implementations. In order to facilitate the discussion and to drive the reader toward the main goal of this work (i.e. showing the potentialities of the hybrid design with respect to the conventional MOSFET and full-TFET design strategies), only the results related to the topology proposed in [20] will be initially discussed in detail, focusing on the specific features of the three possible device implementations (i.e. MOSFET, full-TFET and mixed TFET-MOSFET); the impact of device variability will be presented too. Afterwards, the potentiality of the mixed solution will be investigated in the full range of topologies discussed so far, in order to show that the achievements are mostly independent on the particular circuit topology.

\section{A. Dynamic figures of merit}

Fig.5 illustrates the transient behavior of the LS circuit initially proposed in [20]. It is easy to observe that when TFETs are used to replace MOSFETs driven by the lowvoltage signals, the current contention at discharging internal nodes (either Q1 or Q2) is greatly reduced due to the higher switching currents carried by the TFETs which cause the positive feedback to be triggered faster. Note that the switching speed of the pure TFET solution is favored by the increased drive strength of the pull-down networks (PDNs) in discharging internal nodes (as occurs for the mixed TFETMOSFET circuit) but, at the same time, this effect is in part jeopardized by the slower rising voltage transition on the charging internal node due to the reduced drive strength of the pTFET-based PUNs.

It is worth pointing out that the faster switching allows for a significant reduction of the short-circuit current (and consequently energy consumed per transition) occurring during the change of the state of the LS. This is particularly

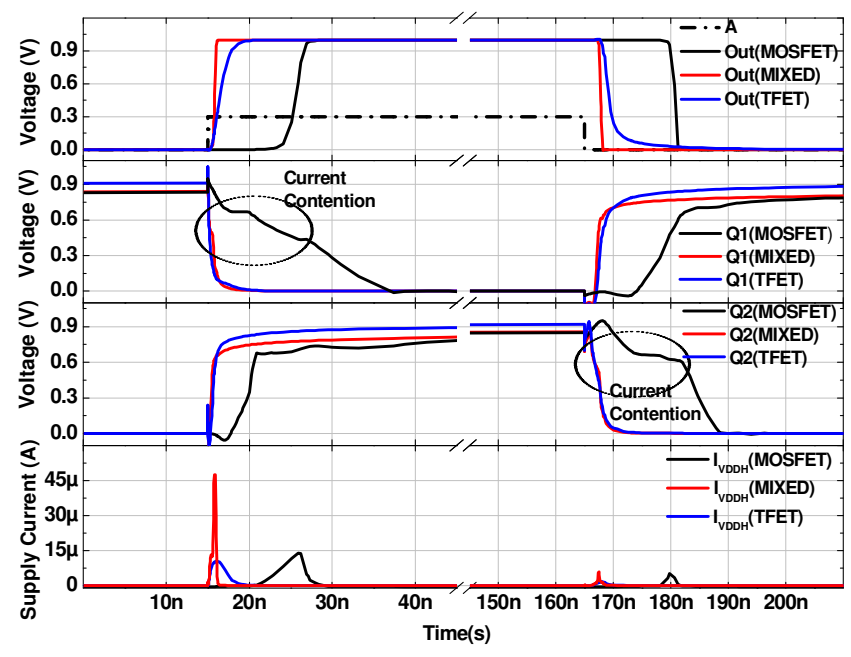

Fig.5. Transient behavior of the circuit in [20] (Fig.4c).

crucial when the input signal has slow slew rate and ultralow voltage level.

The impact on energy and delay of the conventional MOSFET, pure TFET and mixed TFET-MOSFET options is better quantified in Fig.6 (data are given in Table I). In this analysis, $\mathrm{V}_{\mathrm{DDL}}$ ranges from the deep sub-threshold regime to $0.6 \mathrm{~V}$, whereas the $\mathrm{V}_{\mathrm{DDH}}$ is fixed to $1 \mathrm{~V}$.

The higher switching current and the better SS of TFETs considerably improve the speed of the TFET-based circuits for the lower input voltages. As an example, for a $0.2 \mathrm{~V}$ input signal the pure TFET solution reduces the delay more than $6 \mathrm{X}$ in comparison to the conventional MOSFET design. For the same voltage conversion operation, the hybrid design, which benefits of both the merits of TFETs (i.e. strengthened PDNs) and MOSFETs (faster triggering of the positive feedback in the PUNs), achieves even higher speed advantages, thus resulting to be faster than $2 \mathrm{X}$ and $12.5 \mathrm{X}$ in comparison to the pure TFET and conventional MOSFET implementations, respectively. Note also that the effective voltage range for improved speed of the mixed TFET-MOSFET LS is broadened in comparison to the only-TFET solution. Only when the input voltage level becomes higher than $\sim 0.4 \mathrm{~V}$ the conventional MOSFET solution attains the best dynamic performance.

TFET-based solutions also achieve better energy results for $\mathrm{V}_{\text {DDL }}$ up to about $0.4 \mathrm{~V}$, mainly due to the reduced short circuit current. The weaker PUNs of the pure TFET design are favorable from the energy point of view when the input voltage level is extremely low. On the contrary, as the input voltage level increases above approximately $0.3 \mathrm{~V}$, the weaker PUNs negatively impact the short circuit current occurring during the switching of the LS. The hybrid solution exhibits energy consumption close to the minimum over the whole considered input voltage range.

Fig.6c shows that the combined reduction in terms of delay and energy leads the mixed TFET-MOSFET circuit to achieve the best Energy-Delay-Product (EDP) results for $\mathrm{V}_{\mathrm{DDL}}$ less than $\sim 0.4 \mathrm{~V}$ (i.e. about three orders of magnitude at $\mathrm{V}_{\mathrm{DDL}}=$ 

IEEE. Personal use is permitted, but republication/redistribution requires IEEE permission. See http://www.ieee.org/publications_standards/publications/rights/index.html for more information.

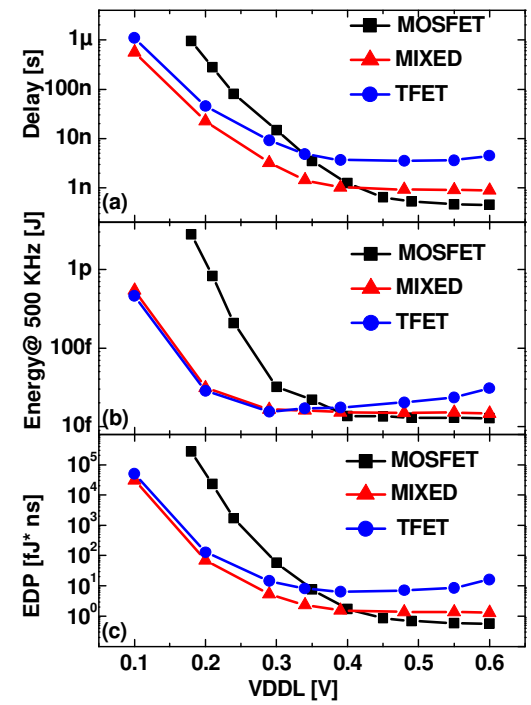

Fig.6. Dynamic performance versus $\mathrm{V}_{\mathrm{DDL}}\left(@ \mathrm{~V}_{\mathrm{DDH}}=1 \mathrm{~V}\right)$ for the LS proposed in [20] (Fig.4c): (a) worst case delay, (b) average energy-pertransition (@ $500 \mathrm{kHz}$ ) and (c) energy-delay product.

$0.2 \mathrm{~V})$, whereas, for higher input voltage levels, the MOSFET design shows only marginal EDP improvements over the mixed solution (less than $5 \%$ at $\mathrm{V}_{\mathrm{DDL}}=0.6 \mathrm{~V}$ ).

\section{B. Impact of device variability}

Work-function variation (WFV), the Line Edge Roughness (LER) and the Random Dopant Fluctuations (RDF) are considered as the leading sources of variability for both MOSFET and TFET devices [12, 23-26]. Due to computational time and resource constraints, a rigorous variability analysis at both device and circuit level is essentially unfeasible, because it would require the generation of a statistically meaningful number of device instances through the TCAD simulator (and the corresponding LUTs for the $\mathrm{I}-\mathrm{V}$ and $\mathrm{C}-\mathrm{V}$ curves) to be used in Monte Carlo (MC) simulations performed at the circuit level. Thus, in our work we consider only WFV. The effect of this source of variability is projected on dynamic and static characteristics of the circuits by 150 runs MC simulations, performed modeling the threshold voltage $\left(\mathrm{V}_{\mathrm{th}}\right)$ variation with a gaussian distribution with a standard deviation obeying to the Pelgrom's law, where $\mathrm{A}_{\mathrm{Vth}}=1 \mathrm{mV} \cdot \mu \mathrm{m}$ was assumed [27].

The variation on $V_{\text {th }}$ has been modeled at the circuit simulator level, by adding a DC voltage generator in series with the gate of each transistor, whose voltage is randomly determined at any run of the MC simulations. This allows one to use only the Verilog-A LUTs with the characteristics of the nominal devices (i.e. only one set of $\mathrm{I}_{\mathrm{D}}-\mathrm{V}_{\mathrm{GS}}-\mathrm{V}_{\mathrm{DS}}, \mathrm{C}_{\mathrm{GD}}-\mathrm{V}_{\mathrm{GS}}-\mathrm{V}_{\mathrm{DS}}$ and $\mathrm{C}_{\mathrm{GS}}-\mathrm{V}_{\mathrm{GS}}-\mathrm{V}_{\mathrm{DS}}$ for any device type), since the considered variability effect is a pure horizontal shift in $\mathrm{V}_{\mathrm{GS}}$ of the characteristics. This is a simplified view of WFV, since more accurate models require the generation of sample devices with random distribution of gate metal grains [23-25]. As said above, this is not feasible for complex circuits using LUTs.
Fig.7 compares the normalized spreads of EDP and static power for MOSFET, mixed and pure TFET configurations. The MOSFET circuit exhibits lower variability in terms of static behavior (static power) and higher variability in terms of dynamic behavior (EDP) with respect to pure TFET and mixed circuits. As reported in Table II, the larger EDP spread of the MOSFET implementation is a consequence of higher variability of the dynamic figures of merit: energy and delay. The observed different variability of static and dynamic parameters can be ascribed mainly to the different normalized slope of the $\mathrm{I}_{\mathrm{D}}-\mathrm{V}_{\mathrm{GS}}$ characteristics $\left(\mathrm{g}_{\mathrm{m}} / \mathrm{I}_{\mathrm{D}}\right.$, where $\mathrm{g}_{\mathrm{m}}=$ $\mathrm{dI}_{\mathrm{D}} / \mathrm{dV}_{\mathrm{GS}}$ ) of MOSFETs and TFETs. From the transfer characteristics in Fig.2a-b, it is evident that TFETs exhibit a higher $\mathrm{g}_{\mathrm{m}} / \mathrm{I}_{\mathrm{D}}$ than MOSFET counterparts at $\mathrm{V}_{\mathrm{GS}}$ close to 0 (corresponding to the better SS) and a lower $\mathrm{g}_{\mathrm{m}} / \mathrm{I}_{\mathrm{D}}$ than MOSFETs for $\mathrm{V}_{\mathrm{GS}}$ higher than about $300 \mathrm{mV}$. We thus infer that the higher variability of static parameters and the lower variability of dynamic parameters are intrinsic properties of all TFET-based circuits and not specific properties of the LS topology.

\section{Impact of the circuit topology}

In this subsection, the impact of different circuit topologies is evaluated considering both mixed TFET-MOSFET and conventional MOSFET solutions. Since the pure TFET implementation has never proved to be the most competitive solution in the above-analyzed operating conditions, it was excluded from the comparative analysis described in the following.

Table I shows the characteristics of the analyzed LS designs for voltage up-conversion from $0.3 \mathrm{~V}$ to $1 \mathrm{~V}$ of a $500 \mathrm{kHz}$ input pulse. In this operating condition, the mixed TFETMOSFET solutions perform significantly better than their conventional MOSFET counterparts. More precisely, the delay is reduced from 5.7 times (for the circuit proposed in [20]) to 7.2 times (for the LS described in [18]). At the same time, energy per operation is more than halved for almost all the circuit topologies. In addition, slight improvements in terms of stand-by power are observed. Interestingly, the significantly higher driving current of TFETs allows for a reduction of the minimum voltage level $\mathrm{V}_{\mathrm{DDL}}{ }^{\min , 500 \mathrm{kHz}}$ (evaluated as the minimum voltage allowing a successful up-conversion to $1 \mathrm{~V}$ of a $500 \mathrm{kHz}$ input pulse) in the deep sub-threshold domain.

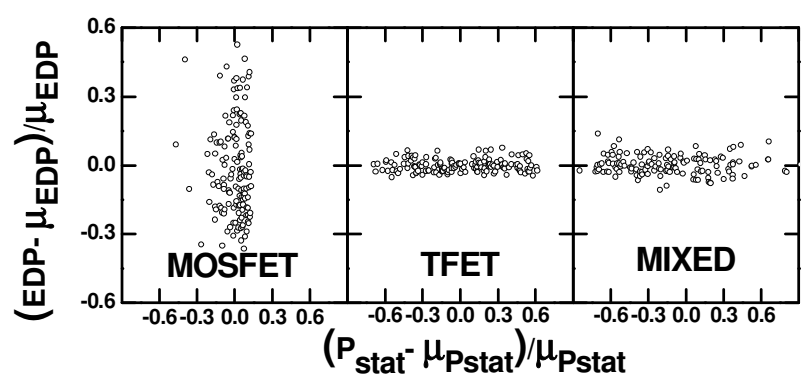

Fig.7. EDP- $\mathrm{P}_{\text {stat }}$ plots from Monte Carlo simulations for $\mathrm{V}_{\mathrm{DDH}}=1 \mathrm{~V}$ and $\mathrm{V}_{\mathrm{DDL}}=0.3 \mathrm{~V}$, considering the LS proposed in [20] (Fig.4c). 

IEEE. Personal use is permitted, but republication/redistribution requires IEEE permission. See http://www.ieee.org/publications_standards/publications/rights/index.html for more information.

TABLE I

FIGURES OF MERIT FOR DIFFERENT LS TOPOLOGIES $\left(\mathrm{V}_{\mathrm{DDH}}=1 \mathrm{~V}, \mathrm{~V}_{\mathrm{DDL}}=0.3 \mathrm{~V}, \mathrm{FREQ}=500 \mathrm{KHZ}\right)$

\begin{tabular}{lccccc}
\hline \hline \multicolumn{1}{c}{ Design } & $\begin{array}{c}\text { Delay } \\
{[\mathrm{ns}]}\end{array}$ & $\begin{array}{c}\text { Energy } \\
{[\mathrm{fJ}]}\end{array}$ & $\begin{array}{c}\mathrm{EDP} \\
{[\mathrm{fJ} * \mathrm{~ns}]}\end{array}$ & $\begin{array}{c}\mathrm{I}_{\text {leak }} \\
{[\mathrm{pA}]}\end{array}$ & $\begin{array}{c}\mathrm{V}_{\mathrm{DDL}}{ }^{\min , 500 \mathrm{kHz}} \\
{[\mathrm{mV}]}\end{array}$ \\
\hline [18] MOSFET & 12.9 & 45.2 & 583.1 & 36.7 & 160 \\
{$[18]$ MIXED } & 1.8 & 18.4 & 33.1 & 30.8 & 70 \\
\hline$[19]$ MOSFET & 13.8 & 68 & 938.4 & 130 & 140 \\
{$[19]$ MIXED } & 2.2 & 15.3 & 33.6 & 13.1 & 120 \\
\hline [20] MOSFET & 18.1 & 32.1 & 581 & 10.3 & 180 \\
{$[20]$ MIXED } & 3.2 & 16.5 & 52.8 & 6.01 & 100 \\
\hline [21] MOSFET & 19.3 & 24.4 & 470.9 & 6.18 & 170 \\
{$[21]$ MIXED } & 2.5 & 12.9 & 32.2 & 6.35 & 110 \\
\hline \hline
\end{tabular}

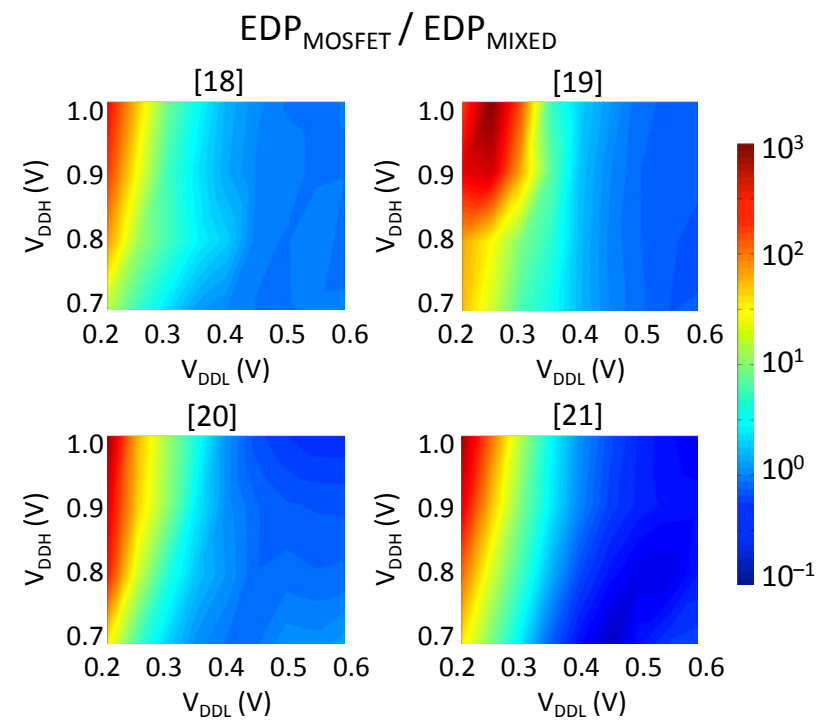

Fig.8. EDP of MOSFETs vs. mixed solution as a function of $\mathrm{V}_{\mathrm{DDH}}$ and $\mathrm{V}_{\text {DDL. }}$

Fig.8 shows the EDP ratio between MOSFET and mixed circuits in the entire voltage range investigated in this work, that is $\mathrm{V}_{\mathrm{DDL}}$ ranging from $0.2 \mathrm{~V}$ to $0.6 \mathrm{~V}$ and $\mathrm{V}_{\mathrm{DDH}}$ ranging from $0.7 \mathrm{~V}$ to $1 \mathrm{~V}$. Due to significant reduction in terms of both delay and energy, the mixed solutions improve the EDP parameter up to two orders of magnitude for lower $\mathrm{V}_{\mathrm{DDL}}$ and higher $\mathrm{V}_{\mathrm{DDH}}$ with only negligible losses for higher $\mathrm{V}_{\mathrm{DDL}}$ and lower $\mathrm{V}_{\mathrm{DDH}}$ (i.e. when the required voltage conversion ratio $\mathrm{V}_{\mathrm{DDH}} / \mathrm{V}_{\mathrm{DDL}}$ is significantly reduced).

Table II provides MC comparison data obtained using the above described simulation methodology. From the given results, the dynamic parameters of the mixed TFET-MOSFET circuits appear to be more robust against $\mathrm{V}_{\text {th }}$ random variations than those of the correspondent MOSFET solutions, at the expense of higher leakage variability. This result is in agreement with the arguments discussed in Section IV-B.

Table III reports the impact of the $\mathrm{V}_{\text {th }}$ variability on the minimum $V_{D D L}\left(V_{D D L}{ }^{m i n}\right)$ that can be successfully upconverted to $1 \mathrm{~V}$. To evaluate $\mathrm{V}_{\mathrm{DDL}}{ }^{\text {min }}$, we set $\mathrm{V}_{\mathrm{DDH}}$ to $1 \mathrm{~V}$,
TABLE II

VARIABILITY RESULTS FOR THE VARIOUS LS TOPOLOGIES $\left(\mathrm{V}_{\mathrm{DDH}}=1 \mathrm{~V}, \mathrm{~V}_{\mathrm{DDL}}=0.3 \mathrm{~V}\right.$, FREQ $\left.=500 \mathrm{KHZ}\right)$

\begin{tabular}{lccccccccc}
\hline \hline \multirow{2}{*}{ Design } & \multicolumn{4}{c}{ Delay } & \multicolumn{4}{c}{ Energy } & \multicolumn{4}{c}{$\mathrm{I}_{\text {leak }}$} \\
\cline { 2 - 10 } & $\begin{array}{c}\sigma \\
{[\mathrm{ns}]}\end{array}$ & $\sigma \mathrm{ns}]$ & $\sigma / \mu$ & $\begin{array}{c}\mu \\
{[\mathrm{fJ}]}\end{array}$ & $\begin{array}{c}\sigma \\
{[\mathrm{fJ}]}\end{array}$ & $\sigma / \mu$ & $\begin{array}{c}\mu \\
{[\mathrm{pA}]}\end{array}$ & $\begin{array}{c}\sigma \\
{[\mathrm{pA}]}\end{array}$ & $\sigma / \mu$ \\
\hline [18] MOSFET & 13.8 & 3.5 & 0.25 & 47.3 & 8.9 & 0.19 & 16.4 & 4.6 & 0.28 \\
[18] MIXED & 2.5 & 0.2 & 0.08 & 18.7 & 0.7 & 0.04 & 11.3 & 4.4 & 0.39 \\
\hline [19] MOSFET & 14.3 & 3.1 & 0.22 & 90.4 & 7.9 & 0.09 & 144 & 50 & 0.35 \\
[19] MIXED & 2.3 & 0.4 & 0.17 & 15.6 & 0.9 & 0.06 & 14.9 & 7 & 0.47 \\
\hline [20] MOSFET & 18.9 & 3.1 & 0.16 & 32.2 & 3.5 & 0.11 & 8.6 & 0.9 & 0.10 \\
[20] MIXED & 3.2 & 0.1 & 0.03 & 15.9 & 0.3 & 0.02 & 8.7 & 5.5 & 0.63 \\
\hline [21] MOSFET & 19.5 & 3.7 & 0.19 & 25.6 & 2.8 & 0.11 & 4.5 & 0.5 & 0.11 \\
[21] MIXED & 1.8 & 0.1 & 0.06 & 12.7 & 0.2 & 0.02 & 3.3 & 0.6 & 0.18 \\
\hline \hline
\end{tabular}

TABLE III

MC ANALYSIS OF THE MINIMUM $\mathrm{V}_{\text {DDL }}$ FOR SUCCESSFUL UP-CONVERSION TO $1 \mathrm{~V}$

\begin{tabular}{lccc}
\hline \hline \multicolumn{1}{c}{ Design } & $\begin{array}{c}\mu \\
{[\mathrm{mV}]}\end{array}$ & $\begin{array}{c}\sigma \\
{[\mathrm{mV}]}\end{array}$ & $\begin{array}{c}\mu+3 \sigma \\
{[\mathrm{mV}]}\end{array}$ \\
\hline [18] MOSFET & 46.7 & 17.3 & 98.5 \\
[18] MIXED & 29.3 & 10.0 & 59.2 \\
\hline [19] MOSFET & 46.2 & 22.1 & 112.6 \\
[19] MIXED & 40.5 & 20.7 & 102.7 \\
\hline [20] MOSFET & 52.4 & 22.0 & 118.4 \\
[20] MIXED & 34.4 & 16.2 & 83.1 \\
\hline [21] MOSFET & 45.5 & 23.4 & 115.6 \\
[21] MIXED & 44.1 & 19.0 & 101.0 \\
\hline \hline
\end{tabular}

swept $\mathrm{V}_{\mathrm{DDL}}$, and measured the minimum input voltage level that produced a correct up-conversion to $\mathrm{V}_{\mathrm{DDH}}$, for each $\mathrm{MC}$ run [28]. It can be easily seen that the MIXED LS designs always allow dependable level up-conversion from lower $\mathrm{V}_{\text {DDL }}$ voltage compared to their MOSFET counterparts (i.e. the extreme $\mu+3 \sigma \quad V_{\text {DDL }}$ value is always lower for MIXED designs).

In this paper, we have benchmarked circuit topologies and design strategies focusing on energy and performance figures of merit without taking into account the area occupancy. For the sake of clarity, we want to point out that the fabrication of TFET devices is still immature, so that layout rules do not exist yet. It is thus very difficult to give a quantitative estimation of the area of the investigated circuits. In this respect, we have used devices with the same length and width to make the comparison as fair as possible. However, the integration of the mixed TFET/MOSFET implementations is likely to result in an area penalty with respect to the full MOSFET and pure TFET implementations, due to the need for a larger number of wells to accommodate devices implemented exploiting different materials.

\section{CONCLUSIONS}

This device-circuit co-design study demonstrates the potentialities of TFET device for the implementation of LS for voltage up-conversion from the ultralow voltage regime. We 
(c) 2015 IEEE. Personal use of this material is permitted. Permission from IEEE must be obtained for all other users, including reprinting/ republishing this material for advertising or promotional purposes, creating new collective works for resale or redistribution to servers or lists, or reuse of any copyrighted components of this work in other works. DOI: 10.1109/TED.2015.2494845 - ๑ 2015 IEEE. Personal use is permitted, but republication/redistribution requires IEEE permission. See http://www.ieee.org/publications_standards/publications/rights/index.html for more information.

propose a mixed TFET-MOSFET strategy of LS design, based on the introduction of TFET devices in the low supply circuit sections. The mixed LS exhibits superior dynamic performance at the same static power consumption than conventional MOSFET and pure TFET solutions. Compared to conventional MOSFET implementation, the mixed solution offers an improvement of the energy delay product up to 3 decades and allows minimum voltage level for successful upconversion to be extended towards significantly lower values. The hybrid LS exhibits lower variability of dynamic parameters, but higher variability in terms of static behavior with respect to conventional MOSFET counterpart. The study on four different topologies confirms that the design of LSs for voltage up-conversion from the deep-voltage regime is intrinsically suitable for a mixed TFET-MOSFET implementation.

\section{REFERENCES}

[1] H. Lu and A. Seabaugh, "Tunnel field-effect transistors: State-of-theart", IEEE Journal of the Electron Devices Society, vol. 2, no. 4, pp. 44 49, 2014.

[2] L. De Michielis, L. Lattanzio, A.M. Ionescu, "Understanding the Superlinear Onset of Tunnel-FET Output Characteristic", IEEE Electron Device Letters, vol. 33, no. 11, pp. 1523-1525, Nov. 2012.

[3] E. Gnani, S. Reggiani, A. Gnudi, G. Baccarani, "Drain-conductance optimization in nanowire TFETs", Proceedings of the European SolidState Device Research Conference (ESSDERC) 2012, pp.105-108, 1721 Sept. 2012.

[4] M. Luisier, G. Klimeck, "Performance comparisons of tunneling fieldeffect transistors made of InSb, Carbon, and GaSb-InAs broken gap heterostructures", IEEE International Electron Devices Meeting (IEDM) 2009, pp. 1-4, 7-9 Dec. 2009.

[5] Sung Hwan Kim, S. Agarwal, Z.A. Jacobson, P. Matheu, Chenming Hu, Tsu-Jae King Liu, "Tunnel Field Effect Transistor With Raised Germanium Source", IEEE Electron Device Letters, vol.31, no.10, pp.1107-1109, Oct. 2010.

[6] Yin-Nien Chen, Ming-Long Fan, V.P.-H. Hu, Pin Su, Ching-Te Chuang, "Design and Analysis of Robust Tunneling FET SRAM", IEEE Transactions on Electron Devices, vol. 60, no. 3, pp. 1092-1098, March 2013.

[7] E. Baravelli, E. Gnani, A. Gnudi, S. Reggiani, G. Baccarani, "TFET Inverters With n-/p-Devices on the Same Technology Platform for LowVoltage/Low-Power Applications", IEEE Transactions on Electron Devices, vol. 61, no. 2, pp. 473-478, Feb. 2014.

[8] D. Esseni, M.Guglielmini, B. Kapidani, T. Rollo, M. Alioto, "Tunnel FETs for Ultralow Voltage Digital VLSI Circuits: Part I-DeviceCircuit Interaction and Evaluation at Device Level", IEEE Transactions on Very Large Scale Integration (VLSI) Systems, vol. 22, no. 12, pp. 2488-2498, Dec. 2014.

[9] D. H. Morris, U. E. Avci, R. Rios, I. A. Young, "Design of Low Voltage Tunneling-FET Logic Circuits Considering Asymmetric Conduction Characteristics", IEEE Journal on Emerging and Selected Topics in Circuits and Systems, vol. 4, no. 4, pp. 380-388, Dec. 2014.

[10] Yin-Nien Chen, Ming-Long Fan, V.P.-H. Hu, Pin Su, Ching-Te Chuang, "Evaluation of Stability, Performance of Ultra-Low Voltage MOSFET, TFET, and Mixed TFET-MOSFET SRAM Cell With WriteAssist Circuits", IEEE Journal on Emerging and Selected Topics in Circuits and Systems, vol. 4, no. 4, pp. 389-399, Dec. 2014.

[11] S. Strangio, P. Palestri, D. Esseni, L. Selmi, F. Crupi, S. Richter, QingTai Zhao, S. Mantl, "Impact of TFET Unidirectionality and Ambipolarity on the Performance of 6T SRAM Cells", IEEE Journal of the Electron Devices Society, vol. 3, no. 3, pp. 223-232, May 2015.

[12] U. E. Avci, D. H. Morris, I. A. Young, "Tunnel Field-Effect Transistors: Prospects and Challenges", IEEE Journal of the Electron Devices Society, vol. 3, no. 3, pp. 88-95, May 2015.

[13] Ming-Long Fan; V.P.-H. Hu, Yin-Nien Chen, Chih-Wei Hsu, Pin Su, Ching-Te Chuang, "Investigation of Backgate-Biasing Effect for
Ultrathin-Body III-V Heterojunction Tunnel FET', IEEE Transactions on Electron Devices, vol. 62, no. 1, pp. 107-113, Jan. 2015.

[14] J.C. Chi, H.H. Lee, S.H. Tsai and M.C. Chi, "Gate level multiple supply voltage assignment algorithm for power optimization under timing constraint", IEEE Transactions on Very Large Scale Integration System, vol. 15, no. 6, pp. 637-648, 2007.

[15] K. Craig, Y. Shakhsheer, S. Arrabi, S. Khanna, J. Lach, and B. H. Calhoun, "A 32 b $90 \mathrm{~nm}$ processor implementing panoptic DVS achieving energy efficient operation from sub-threshold to high performance", IEEE Journal of Solid-State Circuits, vol. 49, no. 2, pp. 545-552, Feb. 2014.

[16] M. Lanuzza, P. Corsonello, S. Perri, "Low-Power Level Shifter for Multi-Supply Voltage Designs", IEEE Transactions on Circuits and Systems II, vol. 59, no. 12, pp. 922-926, Dec. 2012.

[17] S. Lütkemeier, U. Rückert, "A Subthreshold to Above-Threshold Level Shifter Comprising a Wilson Current Mirror", IEEE Transactions on Circuits and Systems II, vol. 57, no. 9, pp. 721-724, Sept. 2010.

[18] W. Zhao, A. Alvarez, Y. Ha, "A 65-nm 25.1-ns 30.7-fJ Robust Subthreshold Level Shifter with Wide Conversion Range", IEEE Transactions on Circuits and Systems II, In Press, 2015.

[19] S.R. Hosseini, M. Saberi, R. Lotfi, "A Low-Power Subthreshold to Above-Threshold Voltage Level Shifter", IEEE Transactions on Circuits and Systems II, vol. 61, no. 10, pp. 753-757, Oct. 2014.

[20] M. Lanuzza, P. Corsonello, S. Perri, "Fast and Wide Range Voltage Conversion in Multisupply Voltage Designs", IEEE Transactions on Very Large Scale Integration (VLSI) Systems, vol. 23 , no. 2, pp. 388391, Feb. 2015.

[21] S.-C. Luo, C.-J. Huang, Y.-H. Chu, "A Wide-Range Level Shifter Using a Modified Wilson Current Mirror Hybrid Buffer", IEEE Transactions on Circuits and Systems I, vol. 61, no. 6, pp. 1656-1665, June 2014

[22] L. Czornomaz, N. Daix, K. Cheng, D. Caimi, C. Rossel, K. Lister, M. Sousa, J. Fompeyrine, "Co-integration of InGaAs n- and SiGe pMOSFETs into digital CMOS circuits using hybrid dual-channel ETXOI substrates", IEEE International Electron Devices Meeting (IEDM) 2013, pp. 2.8.1-2.8.4, 9-11 Dec. 2013.

[23] K. M. Choi et al., "Work-Function Variation Effects of Tunneling FieldEffect Transistors (TFETs)", IEEE Electron Device Letter, vol. 34, no. 8, pp. 942-944, 2013

[24] M.-L. Fan et al., "Analysis of Single-trap-Induced Random Telegraph Noise and Its Interaction With Work Function Variation for Tunnel FET", IEEE Tran. on Electron Device, vol. 60, no. 6, pp. 2038-2044, 2013.

[25] Y. Lee et al., "Study of Work-Function Variation for High-k/Metal-Gate Ge-Source Tunnel Field-Effect Transistors", IEEE Tran. on Electron Device, vol. 62, no. 7, pp. 2143-2147, 2015.

[26] N. Damrongplasit et al., "Impact of Gate Line-Edge Roughness (LER) Versus Random Dopant Fluctuations (RDF) on Germanium-Source Tunnel FET Performance", IEEE Transactions on Nanotechnology, vol.12, no.6, pp.1061-1067, Nov. 2013.

[27] Y.X. Liu, K. Endo, S. O'uchi, T. Kamei, J. Tsukada, H. Yamauchi, Y. Ishikawa, T. Hayashida, K. Sakamoto, T. Matsukawa, A. Ogura, M. Masahara, "On the gate-stack origin threshold voltage variability in scaled FinFETs and multi-FinFETs", Symposium on VLSI Technology (VLSIT) 2010, pp. 101-102, 15-17 June 2010.

[28] S.N. Wooters, B.H. Calhoun and T.N. Blalock, "An energy-efficient subthreshold level converter in 130-nm CMOS", IEEE Trans. on Circuits and Systems II: Express Briefs, vol. 57 (4), pp. 570-578, Apr. 2010 . 